\title{
De la connexité entre l'organisation du travail gouvernemental et la gestion de projet
}

\author{
Charité de Béssombe Betjol Hiol, M. Sc. \\ Administrateur Civil Principal, Chargé d'études \\ Secrétariat Général des Services du Premier Ministre - Cameroun \\ Julien Bousquet, Ph.D. et Christophe Leyrie, D. Sc. \\ Laboratoire d'études multidisciplinaires en gestion de projet \\ Professeurs, Université du Québec à Chicoutimi
}

\section{INTRODUCTION}

\section{Qu'y a-t-il de commun entre l'organisation du travail gouvernemental (OTG) et la gestion de projet?}

Qu'y a-t-il de commun entre l'organisation du travail gouvernemental (OTG) et la gestion de projet? Ces deux concepts semblent si éloignés et différents l'un de l'autre qu'il peut s'avérer osé, voire périlleux, de rechercher une corrélation utile entre eux, du moins sur le plan de la recherche. L'OTG est semblable à une hydre de mer. Tentaculaire et difficile à apprivoiser. Au plan scientifique ou académique, elle fait partie des sciences administratives appliquées et relève de ce fait des sciences sociales. Elle se situe à la croisée de plusieurs disciplines dont le Droit public (Droit constitutionnel et Droit administratif) et la Science politique (Science administrative et Sociologie politique). Même si elle fait l'objet d'enseignements ou de séminaires dans certaines écoles spécialisées, elle relève davantage de la pratique administrative et revêt des caractéristiques à la fois communes et spécifiques aux États.

La gestion de projet quant à elle s'est affirmée au fil des années comme une discipline scientifique à part entière, tant dans le domaine des sciences sociales en général que dans celui des sciences de gestion en particulier. De plus en plus, elle mobilise un corpus de connaissances, des outils et techniques spécifiques. Elle ouvre la voie à des corps de métiers ou à des qualifications professionnelles internationalement reconnues. Apparue pour la première fois dans les sociétés d'ingénierie pétrochimique et d'armement américaines, avant de connaître un essor spectaculaire vers la fin du $\mathrm{XX}^{\mathrm{e}}$ siècle, la gestion de projet s'est considérablement répandue dans le secteur privé. Elle est, à la faveur de la crise du début des années 1990, considérée comme la solution permettant aux entreprises privées, sinon d'optimiser leurs parts de marché, du moins de les conserver, en maîtrisant notamment les coûts et les délais. De nos jours, elle constitue une démarche privilégiée pour piloter l'exécution des projets qui font désormais partie intégrante des activités des organismes publics ou privés ${ }^{1}$.

De prime abord, l'on peut avoir l'impression que la gestion de projet et l'OTG sont diamétralement opposées. Cependant, si l'OTG consiste pour l'essentiel à traduire un programme politique en projets concrets en vue de satisfaire les besoins des populations, les processus que mobilisent les gouvernants à cet effet peuvent-ils, d'une manière ou d'une autre, s'apparenter à ceux utilisés en matière de gestion de projet? En d'autres termes, existe-t-il un lien quelconque entre l'OTG et la gestion de projet? En l'état actuel de la littérature, aucune étude ne renseigne sur les interactions possibles entre ces deux champs théoriques. La présente réflexion vise à explorer la relation qui pourrait s'établir entre le travail gouvernemental et la gestion de projet, dans la perspective d'un enrichissement mutuel de ces deux domaines de connaissance. 


\section{LE CADRE THÉORIQUE}

\subsection{L'organisation du travail gouvernemental et la gestion de projet : aux sources de l'organisation scientifique du travail}

D'une manière générale, l'organisation du travail, en tant que stratégie d'action des entités publiques ou privées, remonte au début du $\mathrm{XX}^{\mathrm{e}}$ siècle. Elle s'abreuve à la théorie de Taylor sur l'organisation scientifique du travail, selon laquelle la rationalisation du travail constitue l'un des moyens essentiels permettant d'optimiser le rendement des entreprises privées. Cette théorie a connu un raffinement singulier grâce à Fayol qui a structuré la pensée rénovatrice de la gestion de l'entreprise autour de six fonctions essentielles. D'après lui, les fonctions verticales ou spécifiques au sein d'une entreprise sont la technique (production, transformation et fabrication), le commercial (achat, vente et échanges), la finance (recherche et utilisation optimale des capitaux), la comptabilité (calcul de la rémunération et statistiques) et la sécurité (protection des personnes et des biens).

Quant à la fonction administrative dont le détenteur est de nos jours appelé «gestionnaire» ou « manager», elle a un caractère horizontal ou transversal. Elle s'articule autour de cinq activités principales: planifier ou anticiper sur le futur; organiser ou mettre à la disposition de l'entreprise les ressources nécessaires à son fonctionnement; commander (qu'il faut entendre aujourd'hui par «animer »), c'est-à-dire indiquer les tâches et instructions à mettre en œuvre; coordonner ou instaurer la cohérence et l'harmonie entre tous les actes d'une entreprise, en vue de faciliter son fonctionnement et garantir son succès; contrôler ou vérifier que tout se passe conformément au programme arrêté, aux ordres donnés, aux principes admis, de manière à identifier et corriger les manquements constatés.

L'organisation scientifique du travail apparaît de ce fait comme un ensemble d'éléments qui interagissent, dans le but d'améliorer l'efficacité du personnel et permettre à l'entreprise d'atteindre ses objectifs. Cette conception du travail, propre aux entreprises privées, s'est progressivement diffusée au sein de l'administration publique, à la faveur de la diffusion des principes du nouveau management public. Fayol va ainsi poser les bases d'un style de management dont les traits caractéristiques ont été réceptionnés dans les domaines de la gestion de projet et du travail gouvernemental, en ce sens qu'ils mobilisent chacun des mécanismes de planification, d'organisation, d'animation, de coordination et de contrôle.

L'organisation scientifique du travail apparaît de ce fait comme un ensemble d'éléments qui interagissent, dans le but d'améliorer l'efficacité du personnel et permettre à l'entreprise d'atteindre ses objectifs.

\subsection{La doctrine du travail gouvernemental et de la gestion de projet}

L'OTG s'articule autour de deux expressions clés dont il convient de saisir la signification: «organisation du travail», d'une part, dont les fondements historiques ont été rappelés dans la section précédente et l'adjectif «gouvernemental », d'autre part. Ce dernier découle du verbe « gouverner », tiré du mot latin gubernare et du grec kubernan, qui signifie « diriger un navire ». Si l'on peut considérer que l'État est un grand navire, gouverner signifie donc diriger l'État ou conduire les affaires de l'État, en suivant un cap en principe prédéfini. Le décryptage scientifique de l'OTG a mobilisé depuis des lustres nombre de chercheurs ${ }^{2}$, même si la littérature en la matière demeure peu dense et le plus souvent indirecte. En effet, le travail gouvernemental est généralement traité de façon incidente dans le cadre de l'analyse des politiques publiques, au point de se confondre parfois à elles. Certains auteurs ${ }^{3}$ soulignent qu'analyser les politiques publiques permet d'interroger, ce que les gouvernements font, pourquoi ils le font et ce que ça change, ou encore tout ce que le gouvernement décide de faire ou de ne pas faire 4 .

Au milieu des années 1980, la littérature ${ }^{5}$ sur l'OTG va s'affiner au point de s'ordonner autour de deux types d'activités : d'une part, une participation au processus de décision, qui consiste à suivre l'élaboration, l'édiction et la mise en œuvre des textes législatifs ou règlementaires qu'implique la réalisation du programme gouvernemental. D'autre part, une fonction d'ordre administratif, visant à 
assurer la coordination des activités et des réunions gouvernementales, ainsi que la gestion des services qui peuvent être rattachés au chef de l'exécutif. Plus précisément, l'OTG se définit comme le processus qui permet de traduire le programme politique dont sont porteurs les membres du Gouvernement en actes de toute nature qu'eux-mêmes ou leurs subordonnés accomplissent dans l'exercice de leurs fonctions pour la mise en œuvre de ce programme ${ }^{6}$. Au total, le travail gouvernemental apparaît comme Janus biface, en ce sens qu'il revêt une double dimension. D'un côté, il a un versant politique et relève par conséquent du niveau stratégique. $\mathrm{Vu}$ sous cet angle, il a pour point d'ancrage le programme politique ou le plan d'actions du Gouvernement, lequel se décline en politiques et action publiques. De l'autre, il a un versant opérationnel et se rapporte à l'action administrative. Celle-ci prend du relief aussi bien dans la coordination du travail gouvernemental que dans l'exécution de la politique conduite par le gouvernement.

En ce qui concerne les notions de projet (ou plutôt de «project») et de gestion de projet, elles se révèlent complexes, protéiformes et quelquefois galvaudées. Théoriciens et praticiens rivalisent de dextérité pour leur donner un contenu qui tienne compte de leurs caractéristiques intrinsèques et extrinsèques. Dès lors, les propositions pour les appréhender fusent. Néanmoins, les définitions recensées dans la littérature intègrent, suivant des terminologies parfois nuancées, deux idées essentielles : la livraison d'un extrant plus ou moins déterminé et le respect d'un certain nombre de contraintes (qualité, coût, délais et contenu). La

\section{LE CADRE CONCEPTUEL}

\subsection{Le projet et son cycle de vie}

\section{L'idée de cycle de vie du projet fait référence à la répétition des phases d'un projet à l'autre et au fait que les leçons tirées de la dernière phase d'un cycle de vie (l'évaluation) sont censées inspirer et influencer les projets futurs.}

Le projet peut se définir comme l'expression d'un désir, d'une volonté, d'une intention, d'une ambition. C'est aussi l'expression d'un besoin, d'une gestion de projet implique une structure temporaire, hautement organique, capable de réagir rapidement, facilitant l'intégration et les communications tant horizontales que verticales. Elle consiste à planifier, organiser, diriger et contrôler les ressources consenties en vue d'accomplir un objet découlant des buts et des objectifs plus vastes ${ }^{7}$.

En ce qui concerne les notions de projet (ou plutôt de «project») et de gestion de projet, elles se révèlent complexes, protéiformes et quelquefois galvaudées.

Cette approche s'inscrit dans la voie tracée par Fayol pour qui le rôle de gestionnaire est défini suivant les fonctions traditionnelles de management à savoir «planifier», « organiser », «décider» et «contrôler», auxquelles il est suggéré d'ajouter la fonction «communiquer», compte tenu de son importance dans la gestion des parties prenantes ${ }^{8}$. En règle générale, tout projet infère un livrable final (produit ou service) tandis que la gestion de projet renvoie au processus de développement de ce livrable suivant des approches susceptibles de varier. Au demeurant, certains auteurs $^{9}$ font remarquer que le modèle de système de projet est polyvalent et adaptable à toute organisation, indépendamment de son champ d'action. L'on comprend donc pourquoi la gestion de projet, jadis l'apanage des entreprises privées, s'est aujourd'hui démocratisée, au point d'irradier des segments d'activités considérés comme étant par nature exclus de son champ d'action originel. C'est le cas de l'organisation du travail gouvernemental.

nécessité, d'une situation future vivement souhaitée. À bien d'égards, tout projet est semblable à un être humain. Il naît, c'est sa conception ou son démarrage. Il vit, c'est son exécution ou sa réalisation. Il meurt, c'est sa clôture ou sa terminaison. L'idée de cycle de vie du projet fait référence à la répétition des phases d'un projet à l'autre et au fait que les leçons tirées de la dernière phase d'un cycle de vie (l'évaluation) sont censées inspirer et influencer les projets futurs.

Dans la littérature, un consensus se dégage sur le fait que le cycle de vie d'un projet, qu'il soit de 
grande ou de petite taille, simple ou complexe, « mou » (soft project) ou « dur» (hard project), est constitué de phases. D'aucuns ${ }^{10}$ soulignent que le cycle de vie d'un projet se décline en quatre phases : la conception et l'évaluation; la planification détaillée; la réalisation, le suivi et le contrôle; l'exploitation et l'évaluation de la performance du projet réalisé. D'autres ${ }^{11}$ propose une méthodologie en cinq phases (conception, décision, action, contrôle et amélioration continue, intégration) pour traduire la mise en place de la stratégie de l'entreprise à travers des projets, dans une optique orientée vers la satisfaction des clients. Malgré ces nuances, la doctrine semble s'être stabilisée d'une manière générale sur un "phasage » structuré autour des points suivants :

- le démarrage ou la conception du projet : cette phase correspond à ce qui devrait se faire. Il s'agit d'identifier et d'analyser les besoins ou attentes des clients que l'on veut satisfaire à travers le projet, de spécifier les procédés et de sélectionner les opportunités d'amélioration. En outre, il est question à ce niveau d'inventorier les axes de solution possibles et de cerner le concept ou contenu du projet qui sera réalisé;

- l'organisation et la préparation ou la définition $d u$ projet: c'est la phase de la décision. Elle correspond à ce qui peut se faire. Ainsi, les contours ou paramètres techniques du projet sont définis et consignés dans les différents plans de management. Les activités à mettre en œuvre sont programmées et des dispositions sont prises en vue du management du projet. Par ailleurs, les projets sont organisés par catégories pour faciliter le processus de prise de décision;

- l'exécution du travail ou la réalisation du projet: elle correspond à la phase de l'action et consiste à faire; c'est-à-dire à exécuter le projet suivant la planification arrêté en mobilisant tous les processus contractuels. L'équipe projet procède ainsi à la mise en vigueur adéquate du plan de compétences défini;

- la clôture ou la terminaison du projet: elle correspond à la phase de contrôle des résultats et d'amélioration continue. Autrement dit, elle consiste à faire mieux et se traduit par l'évaluation du travail exécuté, le transfert du produit du projet au client et la documentation de clôture du projet.

\subsection{Peut-on parler du cycle de vie du travail gouvernemental?}

Eu égard à ce qui précède, l'on constate qu'il existe bel et bien ce que l'on peut appeler « le cycle de vie du travail gouvernemental ».

La littérature ${ }^{12}$ laisse apparaître une structuration en cinq phases des tâches qu'accomplissent les autorités publiques dans le cadre du travail gouvernemental. Ces tâches se déclinent comme suit :

- l'identification d'un problème : c'est la phase où un problème est identifié par le système politique comme exigeant un traitement et il s'inscrit sur l'agenda d'une autorité publique. C'est donc le point de départ ou l'acte de naissance du travail gouvernemental;

- la formulation de solutions : c'est la phase où des réponses sont étudiées, élaborées, négociées pour établir un cours d'action par l'autorité publique. Toutes les solutions possibles au problème identifié sont analysées et comparées sur la base de différents critères, afin de permettre à l'autorité investie du pouvoir de décision de se prononcer en connaissance de cause;

- la prise de la décision: le décideur gouvernemental officiellement habilité choisit une solution particulière qui devient la politique légitime;

- la mise en œuvre du programme : une politique est appliquée et administrée sur le terrain. C'est la phase dite d'implémentation ou de réalisation;

- la terminaison de l'action: c'est la phase de l'évaluation des résultats. Elle intervient au terme du processus de mise en œuvre et est susceptible de déboucher sur un abandon de l'action spécifique.

Eu égard à ce qui précède, l'on constate qu'il existe bel et bien ce que l'on peut appeler « le cycle de vie $\mathrm{du}$ travail gouvernemental». C'est-à-dire un séquençage de phases, à partir du moment où un problème est inscrit sur l'agenda des autorités publiques d'où émergent les premières idées d'actions à conduire en guise de réponse à formuler, jusqu'à l'évaluation des activités menées à cet effet. Dès lors, une comparaison entre le cycle de vie du projet et celui du travail gouvernemental devient envisageable. 
2.3 Comparaison entre le cycle de vie du projet et le cycle de vie du travail gouvernemental

L'analyse comparative du cycle de vie du travail gouvernemental et du cycle de vie du projet révèle des similitudes ou du moins des recoupements, comme l'atteste le tableau ci-dessous (voir tableau 1). L'on observe, comme le diraient les juristes, un parallélisme de forme, de procédure et de fond entre ces deux cycles de vie.
S'agissant du parallélisme de forme, le travail gouvernemental et la gestion de projet sont formalisés en groupes de processus identiquement hétérogènes. Il s'agit des processus de planification ou de conception, des processus d'organisation et de décision, des processus d'exécution ou de réalisation, des processus de contrôle et d'évaluation. Ces processus sont dynamiques, ce qui leur confère une nature cyclique.

\section{Tableau 1 - Comparaison synoptique du cycle de vie du projet et du cycle de vie du travail gouvernemental}

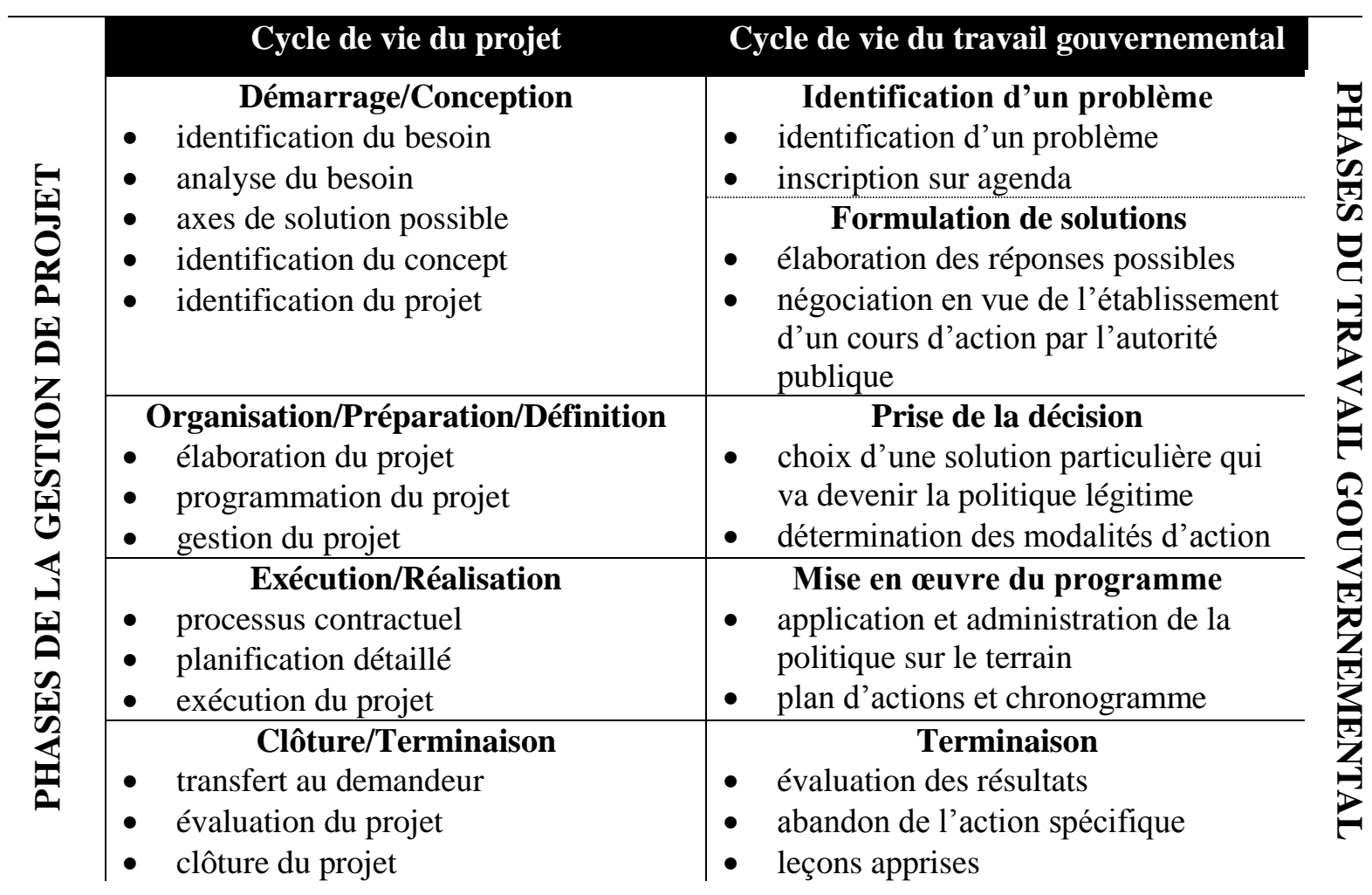

Concernant le parallélisme de procédure, et dans le prolongement des groupes de processus susévoqués, les cycles de vie du projet et du travail gouvernemental s'établissent en phases ou étapes dont la déclinaison se fait en cascades. Ces phases sont bâties suivant une logique verticale ou descendante et s'enchâssent les unes aux autres.

Premièrement, la phase de démarrage ou de conception d'un projet correspond aux phases d'identification du problème et de formulation de solutions dans le cadre du travail gouvernemental. De part et d'autre, tout commence par l'identification d'un besoin ou d'un problème. La volonté des dirigeants d'une organisation donnée (entreprise, État) d'apporter une réponse à ce besoin va les amener à se saisir du problème et à explorer toutes les solutions possibles.

En deuxième lieu, la phase d'organisation ou de définition du projet renvoie à celle de la prise de décision au titre de l'action gouvernementale. C'est le moment où les autorités compétentes s'engagent à agir ou à ne pas agir (go/no go). Le profil du projet ou de la décision résulte du tri sélectif préalablement effectué à partir des différentes options sur table. Troisièmement, la phase de l'exécution du projet correspond à celle 
de la mise en œuvre de la mesure arrêtée. C'est l'application sur le terrain de la décision prise ou la réalisation du projet, suivant les contours et la planification définis. Enfin, la quatrième phase du projet, c'est-à-dire celle de sa clôture, est identique à celle de la terminaison du travail gouvernemental. L'action accomplie est évaluée et documentée pour le futur.

Pour ce qui du parallélisme de fond, il tient aux éléments intrinsèques de chacune des phases des cycles de vie en question. À l'instar de tout projet, le travail gouvernemental n'est pas le fruit du hasard. Il se conçoit et est le résultat d'une démarche cognitive s'inscrivant dans un processus d'idéation et de prise de décisions. Il est alimenté par la nécessité d'apporter des solutions concrètes aux problèmes et aux besoins formulés par une cible donnée. Dans la plupart des cas, les décisions des autorités publiques qu'elles sont imposées (tyranniques) ou négociées (démocratiques), font préalablement l'objet «d'études de faisabilité », fussent-elles sommaires, visant à déterminer les avantages et inconvénients de chaque option.

Ces études peuvent porter sur des aspects politiques, techniques, financiers, économiques, environnementaux, juridiques ou organisationnels. La décision choisie sera ensuite mise en œuvre et évaluée à terme, afin d'en tirer toutes les leçons qui s'imposent. Indubitablement, chaque action constitutive du travail gouvernemental a

\section{CONCLUSION}

\section{En tout état de cause, « l'approche projet » est aujourd'hui une réalité dans le pilotage de l'action publique.}

À partir des développements qui précèdent, trois constats peuvent être faits. D'abord, comme tout projet, l'action gouvernementale a un début et une fin. Chacune de ses phases est constituée d'éléments interreliés. C'est dans ce cycle que s'insère le programme gouvernemental, qui se décline en politiques et en projets publics, par le biais de l'action administrative. Ensuite, l'on observe que des passerelles sont établies entre le cycle de vie du projet et celui du travail une date de début et une date de fin. De même, l'action publique débouche inexorablement sur un produit, qui peut être un bien (un dispensaire construit ou une route), un service (la mise en place d'un système de budgétisation par programme) ou une norme (loi, décret, arrêté, etc.), conformément aux caractéristiques définies en fonction $\mathrm{du}$ besoin exprimé ou ressenti (latent).

De même qu'en gestion de projet, le travail gouvernemental mobilise pour sa mise en œuvre, diverses parties prenantes ayant souvent des intérêts antagonistes et des niveaux d'influence différents, une équipe (il peut s'agir d'une ou plusieurs directions opérationnelles au sein d'une administration donnée, d'un comité ou d'un conseil, etc.), des ressources (humaines, matérielles, logistiques, temporelles et financières). Il est également soumis à des contraintes qu'il appartient aux autorités publiques d'intégrer et à des risques qu'elles doivent anticiper, mesurer et gérer, par l'élaboration et l'application d'une véritable stratégie de mitigation.

\section{De même qu'en gestion de projet, le travail gouvernemental mobilise pour sa mise en œuvre, diverses parties prenantes ayant souvent des intérêts antagonistes et des niveaux d'influence différents.}

gouvernemental. En effet, l'action gouvernementale découle d'une conception, à partir d'un besoin que les autorités publiques décident de satisfaire ou d'un problème qu'elles souhaitent résoudre (fourniture de l'énergie électrique, construction d'un centre de santé, etc.). Elle s'organise au sein des officines administratives, à travers différentes instances de travail (Conseils des ministres ou de cabinet, réunions interministérielles, groupes de travail). Elle se met en œuvre grâce à l'action des agents publics ou se traduit par des projets. À ce titre, elle est soumise à des contraintes et mobilise diverses parties prenantes (ministères, partenaires techniques et financiers, syndicats, associations). À terme, elle 
débouche le plus souvent sur des produits de différente nature (rapports d'études, réglementation, infrastructures diverses).

Enfin, force est de constater que les groupes de processus qui ont cours en gestion de projet (planification organisationnelle, financière et opérationnelle, surveillance et contrôle, etc.) peuvent s'appliquer dans la conduite des activités gouvernementales. En tout état de cause, «l'approche projet » est aujourd'hui une réalité dans le pilotage de l'action publique. Cette connexité entre le travail gouvernemental et la gestion de projet étant établie, il conviendrait dès lors d'approfondir la réflexion dans le sens notamment de voir dans quelle mesure les différentes approches de gestion de projet recensées dans la littérature peuvent contribuer à l'amélioration du travail gouvernemental.

\section{BIBLIOGRAPHIE}

${ }^{1}$ Ezratty, V., Miny, M. (2006). Manager par projets. Principes et méthodes pour réussir. AFNOR.

${ }^{2}$ Lasswell, H.D., Kaplan, A. (1950). Power and Society: A Framework for Political Inquiry. New Haven, Yale University Press.

${ }^{3}$ Kübler, D., de Maillard, J. (2009). Analyser les politiques publiques. Presses universitaires de Grenoble (PUG).

${ }^{4}$ Howlett, M., M. Ramesh (1995). Studying Public Policy. Policy Cycles and Policy Subsystem, Toronto, Oxford University Press.

${ }^{5}$ Wiener, C. (1987). De la coordination administrative à la décision politique : étude comparée de quelques systèmes. Coordination administrative et choix politiques. Revue française d'Administration Publique, $n^{\circ} 42$, avril-juin 1987, Institut International d'Administration Publique, pp.251-260.

${ }^{6}$ Fournier, J. (1987). Le travail gouvernemental. Collection Amphis-Dalloz, 1987.

${ }^{7}$ Kezner, H. (1992). Project Management A system Approach to planning, scheluding, and controlling, Ed. Van Nostrand Reinhold.

${ }^{8}$ Bolivar, J.G. (2011). Vers une intégration du développement durable en gestion de projet. Comment intégrer les questions d'environnement et du développement durable dans l'ensemble des méthodologies de gestion de projet? Éditions Universitaires Européennes, 2011.

${ }^{9}$ Asquin, A., Falcoz, C., Picq, T. (2005). Ce que manager par projet veut dire, Éd. d'Organisations, Paris, 2005.
${ }^{10} \mathrm{O}$ 'Shaughessy, W. (2006). Guide méthodologique d'élaboration et de gestion de projet. La gestion de projet et le concept de gestion intégrée. Les éditions SMG. 2e édition 2005-2006, Tome 1. Collection Le Management de Projet.

${ }^{11}$ Ben Mlouka, S.K., Ouinniche, H.K. (2011). Du management de projets au management stratégique par projet: les projets au service de la stratégie. Revue Organisations et territoires, volume $20, \mathrm{n}^{\circ} 2$ et 3 , pp. 89-97.

${ }^{12}$ Thoenig, J-C. (1985). Le travail gouvernemental. In Les politiques publiques. Madeleine Grawitz et Jean Leca, Presses Universitaires de France, Dalloz, 1985. 


\section{REVUE TRIMESTRIELLE}

RELATIONS INDUSTRIELLES

Revue trimestrielle bilingue publiée depuis 1945 par le Département des relations industrielles de I'Université Laval

ARTICLES

Understanding Turnover as a Lifecycle Process: The Case of Young Nurses

NANCY CÔTÉ

La diffusion de l'instrumentation de la gestion des compétences en Amérique du Nord depuis David C. McClelland

DOMINIQUE BOUTEILLER ET PATRICK GILBERT

Words at Work:

Constructing a Labour Conflict

YONATAN RESHEF AND CHARLES KEIM

Le transfert des pratiques de flexibilisation

de l'emploi dans une firme multinationale :

le rôle charnière des acteurs locaux des filiales

ARMEL BRICE ADANHOUNME

Three Worlds of Professional and Managerial Staff in France

JENS THOEMMES

Insatisfaction des salariés et des clients en réaction à l'automatisation des caisses d'un hypermarché : de la prise de parole à la défection et à la négligence

AMADOU BA ET DAVID ALIS

Maximizing the Benefits of Internationalization: The Moderating Role of Labour Flexibility HEUNG-JUN JUNG, SUNG-CHUL NOH AND SUN-WOOK CHUNG

\section{INDUSTRIAL RELATIONS}

QUARTERLY REVIEW

A bilingual quarterly published since 1945 by the Industrial Relations Department, Université Laval

\section{RI/IR EN LIGNE}

$\mathrm{RI} / \mathrm{R}$ est disponible en ligne sur le site Erudit :

www.erudit.org/revue/ri

Pour un abonnement institutionnel, contacter Érudit.

Pour consulter les règles de publication ou pour vous abonner, visitez notre site Internet www.riir.ulaval.ca

\section{RI/IR ONLINE}

$\mathrm{RI} / \mathrm{R}$ is available on line on Erudit website at:

\section{www.erudit.org/revue/ri}

For an institutional subscription to digitalized issues, please contact Erudit.

Visit our website for Notes to contributors or to subscribe:

\section{www.riir.ulaval.ca}

\section{RELATIONS INDUSTRIELLES} INDUSTRIAL RELATIONS

Pavillon J.-A.-DeSève

1025, avenue des Sciences-Humaines

Bureau 3129, Université Laval

Québec (Québec) Canada G1V 0A6

TÉLÉPHONE : (418) 656-2468

COURRIEL / E-MAIL :

relat.ind@rlt.ulaval.ca

www.riir.ulaval.ca 